\title{
New implementation of the sector decomposition in FORM
}

Takahiro Ueda* and Junpei Fujimoto

High Energy Accelerator Research Organization (KEK),

1-1 Oho, Tsukuba, Ibaraki 305-0801, Japan.

E-mail: uedat@post.kek.jp junpei@post.kek.jp

\begin{abstract}
Nowadays the sector decomposition technique, which can isolate divergences from parametric representations of integrals, becomes a quite useful tool for numerical evaluations of the Feynman loop integrals. It is used to verify the analytical results of multi-loop integrals in the Euclidean region, or in some cases practically used in the physical region by combining with other methods handling the threshold. In an intermediate stage of the sector decomposition for the multi-loop integrals, one often has to handle enormously large expressions containing tons of terms. The symbolic manipulation system FORM is originally designed to treat such huge expressions and has a strong advantage for it. In this talk, the implementation of the sector decomposition algorithm in FORM is discussed. A number of concrete examples including cases of multi-loop diagrams are also shown.
\end{abstract}

XII Advanced Computing and Analysis Techniques in Physics Research November 3-7, 2008

Erice, Italy

\footnotetext{
* Speaker.
} 


\section{Introduction}

The Large Hadron Collider (LHC) has started its operation, and International Linear Collider (ILC) is planned as a next generation collider. In order to obtain as much information as possible from such experiments, it is undoubtedly important to know the precise predictions from the theory at high energies. This fact pushes forward recent study on the computation of one- and higher loop corrections in the perturbation theory.

To evaluate the loop integrals, one may try to find formulae of them analytically. However, if they have many loop momenta, many external legs, and many kinematic parameters, it becomes an extremely difficult problem. Another approach to the evaluation of the loop integrals is to perform loop integrals by using a numerical integration method. In principle, it is possible to construct a general recipe for computing loop integrals numerically, which is applicable to a wide enough class of the loop integrals. Even if one has an analytical formula for some integral, it is good idea to have another method for computing any integrals numerically, for checking purpose. In practice, to perform loop integrals numerically, one has to handle several singularities: UV, IR divergences, and singularities from physical thresholds.

In this talk, the implementation of the sector decomposition algorithm [1]2 ${ }^{1}$ which can isolate divergences from parametric representation (e.g., Feynman parameters) of integrals, on a symbolic manipulation system FORM [6], is discussed. Then, an application of the numerical extrapolation method on handling physical thresholds [7] after isolating all IR divergences is also discussed.

\section{The sector decomposition for IR divergent loop integrals}

Here we briefly sketch how the sector decomposition algorithm is applied for IR divergent loop integrals, in a easy example. We consider the one-loop scalar massless on-shell box diagram in $D=4-2 \varepsilon$ dimensions. By introducing Feynman parameters and performing the momentum integration, we arrive to

$$
\begin{aligned}
I_{4}^{0 m} & =\int \frac{d^{D} k}{i \pi^{D / 2}} \frac{1}{\left(k^{2}+i 0\right)\left[\left(k+p_{1}\right)^{2}+i 0\right]\left[\left(k+p_{12}\right)^{2}+i 0\right]\left[\left(k+p_{123}\right)^{2}+i 0\right]} \\
& =\Gamma(2+\varepsilon) \int_{0}^{1} d^{4} x \delta\left(1-x_{1234}\right) \frac{1}{\left(-s x_{1} x_{3}-t x_{2} x_{4}-i 0\right)^{2+\varepsilon}}
\end{aligned}
$$

where $s=\left(p_{1}+p_{2}\right)^{2}, t=\left(p_{2}+p_{3}\right)^{2}$, and $p_{i}^{2}=0(i=1, \ldots, 4)$, and we have used abbreviations, $p_{12}=p_{1}+p_{2}, p_{123}=p_{1}+p_{2}+p_{3}$ and $x_{1234}=x_{1}+x_{2}+x_{3}+x_{4}$. This integral has overwrap singularities on edges of the integration region: the denominator of the integrand vanishes when two variables simultaneously go to zero, e.g., $x_{1}, x_{2} \rightarrow 0$. The sector decomposition algorithm can disentangle such overwrap singularities, by splitting integral domain and making variable replacements, iteratively. One can obtain

$$
I_{4}^{0 m} / \Gamma(2+\varepsilon)=2 \int_{0}^{1} d^{3} x\left[x_{1}^{-1-\varepsilon} x_{2}^{-1-\varepsilon} \frac{\left(1+x_{12}+x_{1} x_{2} x_{3}\right)^{2 \varepsilon}+\left(1+x_{1}+x_{2} x_{13}\right)^{2 \varepsilon}}{\left(-s-t x_{3}-i 0\right)^{2+\varepsilon}}\right.
$$

\footnotetext{
${ }^{1}$ See 3$]$ for a review. Currently, available public codes of the sector decomposition are 445 .
} 


$$
\left.+x_{1}^{-1-\varepsilon} \frac{\left(1+x_{12}+x_{1} x_{3}\right)^{2 \varepsilon}}{\left(-s-t x_{2} x_{3}-i 0\right)^{2+\varepsilon}}\right]+(s \leftrightarrow t) .
$$

Note that, in this form, there are no overwrap singularities, and all singularities are factorised as powers of monomials. This can be expanded with respect to $\varepsilon$ as:

$$
I_{4}^{0 m} / \Gamma(2+\varepsilon)=\frac{C_{2}}{\varepsilon^{2}}+\frac{C_{1}}{\varepsilon}+C_{0}+\mathscr{O}(\varepsilon),
$$

with

$$
\begin{aligned}
& C_{2}=4 \int_{0}^{1} d x \frac{1}{(-s-t x-i 0)^{2}}+(s \leftrightarrow t), \\
& C_{1}=-4 \int_{0}^{1} d x \frac{\ln (-s-t x-i 0)}{(-s-t x-i 0)^{2}}-2 \int_{0}^{1} d^{2} x \frac{1}{\left(-s-t x_{1} x_{2}-i 0\right)^{2}}+(s \leftrightarrow t), \\
& C_{0}=2 \int_{0}^{1} d x \frac{\ln ^{2}(-s-t x-i 0)}{(-s-t x-i 0)^{2}}+\int_{0}^{1} d^{2} x\left\{\frac{1}{x_{1}} \frac{-12 \ln \left(1+x_{1}\right)-4 \ln \left(1+x_{1} x_{2}\right)}{\left(-s-t x_{2}-i 0\right)^{2}}\right. \\
&\left.\quad+\frac{-4 \ln \left(1+x_{2}\right)+2 \ln \left(-s-t x_{1} x_{2}-i 0\right)}{\left(-s-t x_{1} x_{2}-i 0\right)^{2}}\right\}+(s \leftrightarrow t) .
\end{aligned}
$$

The coefficients of Laurent expansion $C_{2}, C_{1}$ and $C_{0}$ are now expressed in terms of multidimensional integrals, and in the Euclidean region $(s<0$ and $t<0)$, they can be easily computed.

\section{Implementation of the sector decomposition in FORM}

In an intermediate stage of the sector decomposition for more complicated loop integrals, one needs to handle a lot of terms and very large expression. For example, the number of the generated sub-sectors for the scalar massless on-shell triple box diagram is $\mathscr{O}(10000)$, and then many more terms are produced by the $\varepsilon$-expansion. Most of popular computer algebra systems try to keep the expressions in the physical memory. When the expressions become large, the disk memory is used via the virtual memory manager of the computer, and then a computer algebra system can be extremely slowed down or does not work. Therefore, in practice, there is a limit of the size of expressions in such systems ${ }^{2}$. FORM is designed to treat such huge expressions, which are larger than the available physical memory, by using the disk with less penalties in the performance, and therefore has strong advantage.

Our program uses FORM for symbolic manipulations. From the user input file describing the integral, FORM produces Fortran code for the integrand, after the sector decomposition. The program has been checked by various multi-loop integrals, which include planar and non-planar massless on-shell double boxes up to $\mathscr{O}\left(\varepsilon^{0}\right)$, massless three-loop propagators up to $\mathscr{O}\left(\varepsilon^{2}\right)$, etc. As an example, the numerical result of the planar massless on-shell double box is shown in Table 1

\section{Handling physical thresholds by the numerical extrapolation}

Consider the one-loop box diagram Eq. (2.3) again. If either $s$ or $t$ is not negative and the denominator (or the argument of the logarithm) of the integrand is not positive definite, it can become to zero at some points in the integration region. For such singularities arising from physical

\footnotetext{
${ }^{2}$ To avoid this limit, FIESTA [5] uses a data base manager for storing the expressions on the disk efficiently.
} 


\begin{tabular}{|c|c|c|}
\hline & Result and Error & Elapsed Time \\
\hline$C_{4}$ & $0.20000000661904 \mathrm{E}+01 \pm 0.12485517866572 \mathrm{E}-05$ & $<0.1 \mathrm{~s}$ \\
\hline$C_{3}$ & $-0.60000001069171 \mathrm{E}+01 \pm 0.54096786712553 \mathrm{E}-05$ & $<0.1 \mathrm{~s}$ \\
\hline$C_{2}$ & $-0.49167420522766 \mathrm{E}+01 \pm 0.17982248269768 \mathrm{E}-04$ & $0.3 \mathrm{~s}$ \\
\hline$C_{1}$ & $0.11494738132380 \mathrm{E}+02 \pm 0.85267241358588 \mathrm{E}-04$ & $9.5 \mathrm{~s}$ \\
\hline$C_{0}$ & $0.13801183392483 \mathrm{E}+02 \pm 0.29980115734943 \mathrm{E}-03$ & $134.6 \mathrm{~s}$ \\
\hline
\end{tabular}

Table 1: The numerical result of the planar massless on-shell double box for $s=-1$ and $t=-1$ (CPU: Xeon $51603 \mathrm{GHz}$ ). $C_{i}$ are the coefficients of $1 / \varepsilon^{i}$ (overall $\Gamma(3+2 \varepsilon)$ is excluded). With this parameter set, the integrand is relatively smooth (no strong peaks), and we used DCUHRE [8] as the multidimensional integrator. The goal of relative tolerance of the numerical integration is set as $E_{\text {rel }}=10^{-6}$ for each integral. The boldfaced digits indicate they agree with the analytical values.

thresholds, the contour deformation of Feynman parameters is used in [9]. Actually the combination of the sector decomposition and the contour deformation is applied in some practical calculations [10, 11].

Here we consider another possibility, the numerical extrapolation method [7]. In this method, we put $i \delta$ with a small but finite $\delta$ instead of $i 0$ in the denominator. Then we can compute the integral $I(\delta)$ for a given $\delta$. Calculating the sequence $\left\{I\left(\delta_{k}\right)\right\}$ for $\delta_{k}=\delta_{1} r^{k-1}(k=1,2,3, \ldots$, $0<r<1$ ) and extrapolating them by an adequate method, we can finally obtain the result of the integral in the limit of $\delta \rightarrow 0$.

The numerical result of the massless one-loop on-shell box and the massless one-loop box with two adjacent off-shell legs are shown in Table 2 and 3 , respectively.

\section{Summary}

The implementation of the sector decomposition algorithm on a symbolic manipulation system FORM is discussed. Thanks to FORM's advantage for handling very large expressions, the limitation due to the amount of available physical memory is greatly relaxed. After isolating all IR divergences by the sector decomposition, the numerical extrapolation is used for handling threshold singularities. Several numerical results are also shown.

\section{Acknowledgments}

We would like to thank the members of MINAMI-TATEYA collaboration for discussions.

\section{References}

[1] T. Binoth and G. Heinrich, An automatized algorithm to compute infrared divergent multi-loop integrals, Nucl. Phys. $\mathbf{B 5 8 5}$ (2000) 741 [hep-ph/ 0004013 ].

[2] T. Binoth and G. Heinrich, Numerical evaluation of multi-loop integrals by sector decomposition, Nucl. Phys. B680 (2004) 375 [hep-ph/0305234].

[3] G. Heinrich, Sector Decomposition, Int. J. Mod. Phys. A23 (2008) 1457 [0803.4177 [hep-ph] ]. 


\begin{tabular}{|c|c|c|c|}
\hline & & Result and Error & Elapsed Time \\
\hline$C_{2}$ & $\begin{array}{c}\text { real } \\
\text { imaginary }\end{array}$ & $\begin{aligned}-0.162601626011506 \mathrm{E}-03 & \pm 0.216824649637068 \mathrm{E}-11 \\
0.674151650287041 \mathrm{E}-15 & \pm 0.123815752782391 \mathrm{E}-14\end{aligned}$ & $<0.1 \mathrm{~s}$ \\
\hline$C_{1}$ & $\begin{array}{c}\text { real } \\
\text { imaginary }\end{array}$ & 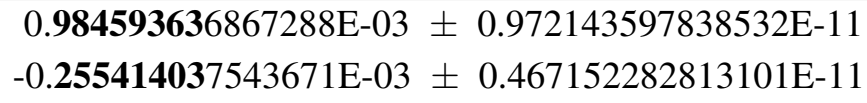 & $0.1 \mathrm{~s}$ \\
\hline$C_{0}$ & $\begin{array}{c}\text { real } \\
\text { imaginary }\end{array}$ & $\begin{aligned}-0 . \mathbf{2 3 8 8 8 0 5} 80536819 \mathrm{E}-02 & \pm 0.327822217718141 \mathrm{E}-10 \\
0 . \mathbf{1 6 0 8 6 7 8 6 6} 223496 \mathrm{E}-02 & \pm 0.159241557716135 \mathrm{E}-10\end{aligned}$ & $0.8 \mathrm{~s}$ \\
\hline
\end{tabular}

Table 2: The numerical result of the massless on-shell one-loop box for $s=123$ and $t=-200$ (CPU: Xeon $51603 \mathrm{GHz}$ ). $C_{i}$ are the coefficients of $1 / \varepsilon^{i}$ (overall $\Gamma(2+\varepsilon)$ is excluded). We used DQAGE in [12], which is one-dimensional integrator, iteratively. The goal of absolute and relative tolerance of the numerical integration are set as $E_{\mathrm{abs}}=10^{-15}$ and $E_{\mathrm{rel}}=10^{-8}$ for each integral. The boldfaced digits indicate they agree with the analytical values.

\begin{tabular}{|c|c|c|c|}
\hline & & Result and Error & Elapsed Time \\
\hline$C_{2}$ & $\begin{array}{c}\text { real } \\
\text { imaginary }\end{array}$ & $\begin{aligned} \mathbf{- 0 . 4 0 6 5 0 4 0 6 5 0 2 8 7 6 6 E -}-04 & \pm 0.542061624092671 \mathrm{E}-12 \\
0.328759384083682 \mathrm{E}-15 & \pm 0.817570959150564 \mathrm{E}-15\end{aligned}$ & $<0.1 \mathrm{~s}$ \\
\hline$C_{1}$ & $\begin{array}{c}\text { real } \\
\text { imaginary }\end{array}$ & $\begin{array}{l}0.341563070020333 \mathrm{E}-03 \pm 0.288030340799012 \mathrm{E}-11 \\
0.127707018334249 \mathrm{E}-03 \pm 0.151057993697844 \mathrm{E}-11\end{array}$ & $0.6 \mathrm{~s}$ \\
\hline$C_{0}$ & $\begin{array}{c}\text { real } \\
\text { imaginary }\end{array}$ & 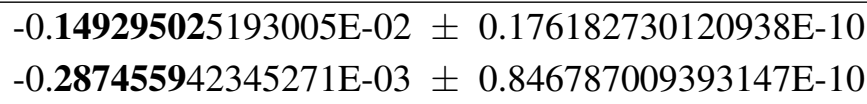 & $1105.9 \mathrm{~s}$ \\
\hline
\end{tabular}

Table 3: The numerical result of the massless one-loop box with two adjacent off-shell legs for $s=123$, $t=-200, p_{3}^{2}=50$ and $p_{4}^{2}=60$. Other conditions are same as Table 2 .

[4] C. Bogner and S. Weinzierl, Resolution of singularities for multi-loop integrals, Comput. Phys. Commun. 178 (2008) 596 [0709.4092 [hep-ph]].

[5] A. V. Smirnov and M. N. Tentyukov, Feynman Integral Evaluation by a Sector decomposiTion Approach (FIESTA), [0807.4129 [hep-ph]].

[6] J. A. M. Vermaseren, New features of FORM, [math-ph/0010025].

[7] E. de Doncker, Y. Shimizu, J. Fujimoto and F. Yuasa, Computation Of Loop Integrals Using Extrapolation, Comput. Phys. Commun. 159 (2004) 145.

[8] J. Berntsen, T. O. Espelid and A. Genz, DCUHRE: an adaptive multidemensional integration routine for a vector of integrals, ACM Trans. Math. Softw. (1991) 452.

[9] Z. Nagy and D. E. Soper, Numerical integration of one-loop Feynman diagrams for N-photon amplitudes, Phys. Rev. D74 (2006) 093006 [hep-ph/ 0610028 ].

[10] A. Lazopoulos, K. Melnikov and F. Petriello, QCD corrections to tri-boson production, Phys. Rev. D76 (2007) 014001 [hep-ph / 0703273$].$

[11] C. Anastasiou, S. Beerli and A. Daleo, Evaluating multi-loop Feynman diagrams with infrared and threshold singularities numerically, JHEP 0705 (2007) 071 [hep-ph / 0703282 ].

[12] R. Piessens, E. de Doncker, C. W. Ubelhuber and D. K. Kahaner, QUADPACK, A Subroutine Package for Automatic Integration, Springer Series in Computational Mathematics. Springer-Verlag, 1983. 